\title{
Overexpression of Gclc in the Drosophila melanogaster thorax
}

\author{
Z. Guvatova ${ }^{1 *}$, M. Shaposhnikov ${ }^{2,4}$, E. Lashmanova ${ }^{3}$, G. Krasnov ${ }^{1}$, \\ A. Kudryavtseva ${ }^{1}$, A. Moskalev ${ }^{1,2,3,4}$ \\ ${ }^{1}$ Engelhardt Institute of Molecular Biology, RAS, Moscow, Russia \\ ${ }^{2}$ Institute of Biology of Komi Science Center of Ural Branch of RAS, Syktyvkar, Russia \\ ${ }^{3}$ Moscow Institute of Physics and Technology, Dolgoprudny, Russia \\ ${ }^{4}$ Syktyvkar State University, Syktyvkar, Russia \\ *e-mail: guvatova.zulfiya@mail.ru
}

Key words: Drosophila melanogaster, gene expression; glutathione, lifespan

Motivation and Aim: The enhancement of glutathione biosynthetic capability can determine longevity and delay aging. Our recent studies demonstrated that Gclc overexpression slows down the age-dependent decline of locomotor activity and circadian rhythmicity without effect on fecundity [1]. Here we analyzed the effects of neuronal Gclc overexpression in the thorax of Drosophila melanogaster on the transcriptomic changes.

Methods and Algorithms: Transcriptomic analysis was performed using control UASGclc flies and flies with Gclc overexpression at the age of 1 (young), 4 (matured) and 6 weeks (old). Processing of transcriptomic data was performed using PPLine toolkit [2] including read preprocessing (trimmomatic), mapping (STAR) and counting (HTSeqcount). The further analysis was done with $\mathrm{R}$ programming language ( $\mathrm{R}$ core Team). The edgeR package was used for analysis of differential expression [3]. KEGG gene set enrichment analysis (GSEA) was performed using clusterProfiler [4].

Results: We derived RNA sequencing expression profiles for 12000 genes (after eliminating low expression ones). The expression of 760 genes $(108$ of 760 have FDR $<0.05)$ demonstrated association with Gclc overexpression in all of the groups: young/mature/ old or males/females. When the selected threshold of expression was 2-fold or more (FDR < 0.05), Gclc overexpression down-regulated 42 genes and up-regulated 14 genes, such as SMC2 (Structural maintenance of chromosomes 2), w (white), CG4293, Gclc, Cyp4p2 (cytochrome P450 4p2), Ipk1 (Inositol-pentakisphosphate 2-kinase), CG8157, CR45457. The Gclc level demonstrated associations with expression of genes involved in a variety of cellular processes.

Conclusion: Transcriptome analysis of the thorax of Gclc transgenic flies revealed pathways that may contribute to the longevity and prevent the age-dependent decline of biological functions.

Acknowledgements: This work costs were funded by the Russian Science Foundation grant No. 14-50-00060.

\section{References}

1. Moskalev A.A. et al. (2016) The influence of pro-longevity gene Gclc overexpression on the agedependent changes in Drosophila transcriptome and biological functions. BMC Genom. 17:273-289.

2. Krasnov G.S. et al. (2015) PPLine: An Automated Pipeline for SNP, SAP, and Splice Variant Detection in the Context of Proteogenomics. J Proteome Res. 14:3729-3737.

3. Robinson M.D., McCarthy D.J., Smyth G.K. (2010) edgeR: a Bioconductor package for differential expression analysis of digital gene expression data. Bioinformatics. 26:139-140.

4. Yu G., Wang L.G., Han Y., He Q.Y. (2012) clusterProfiler: an R package for comparing biological themes among gene clusters. OMICS. 16:284-287. 